\title{
SENSOR PLACEMENT ON SLENDER STRUCTURAL SYSTEMS
}

\author{
J.M. NIEDZWECKI \& S.M. FANG \\ Zachry Department of Civil Engineering, Texas A\&M University, College Station, Texas.
}

\begin{abstract}
There have been many interesting technical ideas and parallels in thinking that have resulted from the technical discussions that I have had with Professor Jerome J. Connor since 1986. This brief article provides a context and reflects upon his mentorship, which helped shape the thinking of a then young professor from another University, and on the impact that Professor Connor has had on his students and others beyond the halls of MIT's Building 1. The optimization topic presented here provides an example of that influence. Extracting the desired response information from an instrumented slender structure, while minimizing the number of sensors, is a challenging problem requiring well-defined objectives that can be used in an optimization process. In this study, a methodology that builds upon a Genetic Algorithm optimization procedure is used to investigate sensor placement needed to recover specific vibration modes. Data recorded from an experiment investigating the flow-induced vibration of a smooth horizontally towed cylinder is used to explore the optimization process and subtleties associated with its application subject to single or multiple objectives and gaps in sensor data due to several possible constraints. The use of the Paterno Front Method and the difficulty in accurately capturing higher modes are addressed.

Keywords: Fiber optic sensors, genetic algorithm, intelligent system design, mode recovery, multiple sensors, optimization, slender structures.
\end{abstract}

\section{INTRODUCTION}

The climate at the Massachusetts Institute in 1986 was very dynamic and focused on the cutting edge topics of artificial intelligence and the development of computer programing environments that could capture and utilize information from a wide range of intellectual pursuits. Seminal textbooks on artificial intelligence and implementations in LISP by Winston [1], the simplest learning machines: prerceptrons by Minsky and Papert [2] and the structure and interpretation of computer programs by Abelson et al. [3] all influenced the direction of future studies in many disciplines. When I arrived at MIT, Professor Connor and his students were deeply involved in the development of new software and concepts regarding intelligent design and computation and new thinking with the potential to impact Civil engineering education and its practice. Figure 1 provides a schematic capturing these ideas.

In the process of exploring the bounds of these ideas and their potential for impact on the analysis and design of structures, several technical articles documenting the thinking during the early stages of this research were written. Areas where this new approach could offer an advantage included the intelligent generation of meshes used in finite element and boundary element methods [4] and the general approach to engineering design [5, 6]. There were many other theses and dissertations at MIT that followed under the direction of Professor Connor and they are archived in the MIT Library. Examples of later studies that were influenced by Professor Connor's vision include later work on the strategy and design of intelligent tutoring systems [7] and preliminary design of deepwater tension leg platforms [8]. 

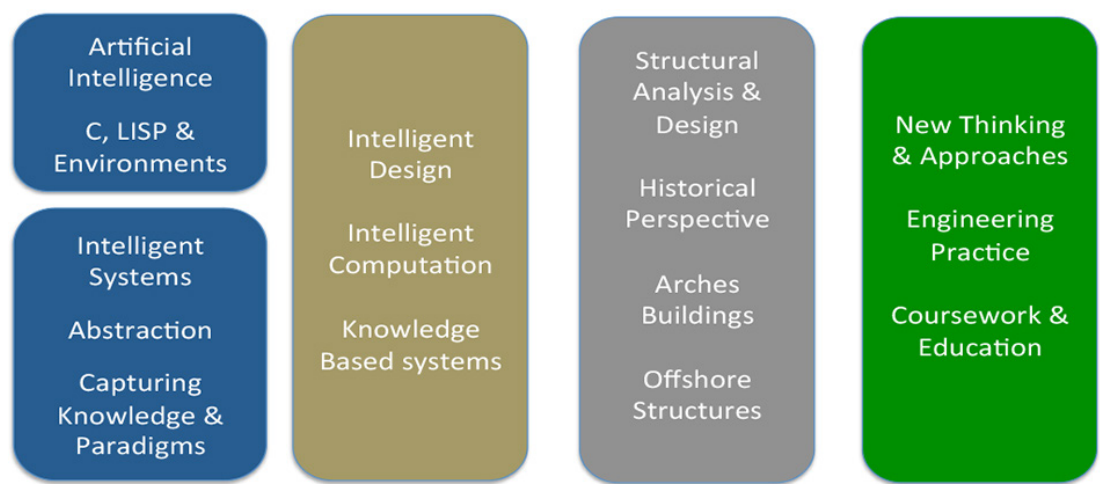

Figure 1: A schematic diagram reflecting the framework, focus, expertise and the breath of research thinking lead by Professor Jerome J. Connor.

More recently, Professor Connor has devoted a great deal of his energy to writing textbooks for structural engineering students and advanced practitioners that reflect his perspective as an exceptional engineering educator and researcher. These recent publications provide a comprehensive view on the fundamentals of structural analyses [9] and capture the originality of the coursework that he has developed on the analysis and control of structural motion [10]. One of his most recent research efforts has been focused on the optimization structural building design. An independent but complementary research investigation addressing optimization strategies of sensors placement on slender structural members and the interpretation of those measurements is addressed in this research paper.

\section{METHODOLOGY}

In determining the appropriate measurement strategy and array of sensors needed to adequately monitor the response behavior of structural systems, many of the same challenges are faced regardless of whether the measurements are to be obtained in a laboratory setting or in the field. It is essential that the measurement objectives be firmly established, so that the attention can be focused on the evaluation and ranking of the various options in order to arrive at and optimal sensor array can be accomplished in an efficient manner. This process typically includes minimizing the number of sensors, addressing sensor placement noting any physical restrictions, the consideration of intentional redundancy for comparative evaluation or to address the potential for sensor failure, the consideration of budget and any other constraints.

Of particular interest in this study is the use of Genetic Algorithms [11], which incorporate the implementation of ideas such as the principle of survival of the fittest and the process of natural selection derived from biological systems. This approach is particularly useful when investigating discontinuous, non-differentiable and nonlinear problems such as sensor optimization for slender structures [12]. A conceptualization of the overall approach and components used in this study is presented in Fig. 2.

The methodology implemented in this study reflects the availability of experimental data from a well-instrumented slender structure experiment that is atypical of most laboratory or field experiments. In those experiments, a large number of fiber optic strain gage sensors were used to measure the flow-induced motions of various towed cylinder configurations $[13,14]$. Some of the bare cylinder data selected from those experiments was selected and the opportunity was provided to explore the recovery of mode shape information using single 


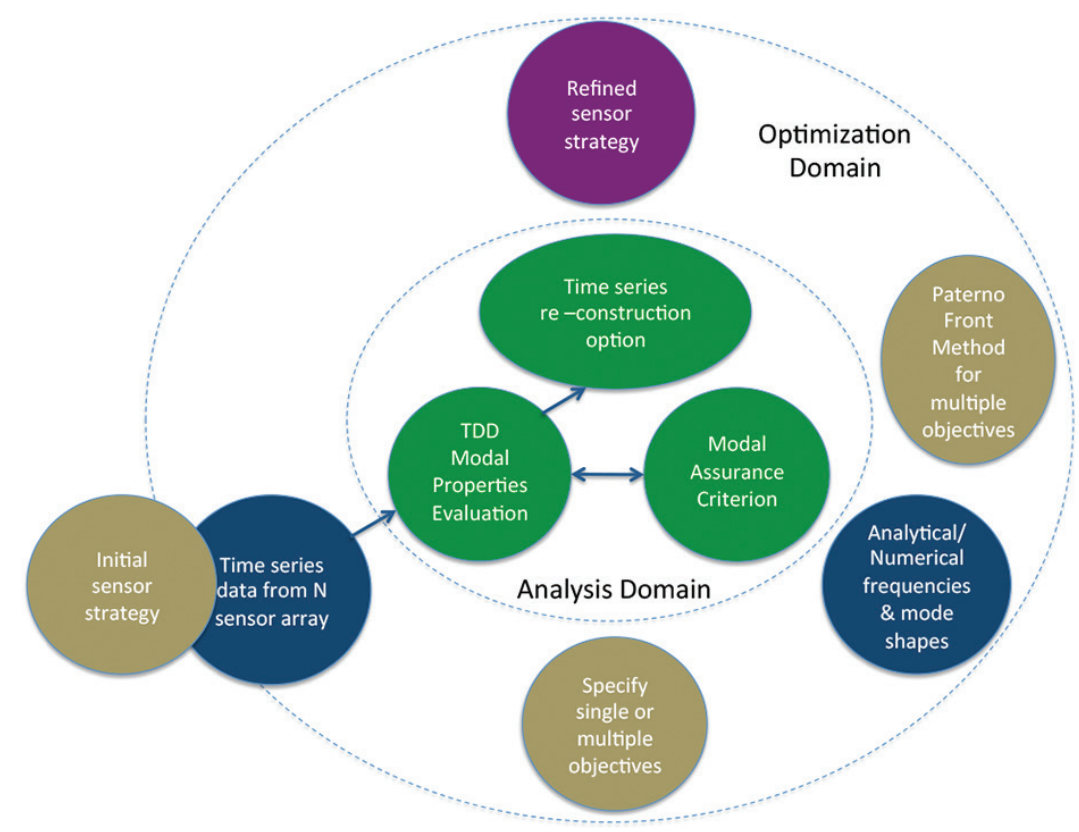

Figure 2: A schematic of the analysis and optimization domain components.

and multiple objective scenarios and restricted sensor placement of sensors. In Fig. 2, experimental data and sensor information is passed into the analysis domain where the time domain decomposition (TDD) method integrated with the modal assurance criteria is used to evaluate the modal properties directly from the data or subset of the data during the optimization process. The resolution of the model properties by the TTD method is directly related to the number of sensors. Details on the use of these techniques for interpreting data from several different data sets can be found in the recent literature [15-17]. An interesting aspect of this analysis approach is the fact that it is possible to check the accuracy of the reconstructed approximation with the original data [18].

As illustrated in Fig. 2, the optimization domain requires additional information prior to the actual optimization process. Specific information that is needed includes specification of the single or multiple objectives to be satisfied, determination of whether analytical or numerical simulations will be integrated into the evaluation procedures and what graphical information will be used to help interpret the optimization results, e.g. Paterno Fronts. So often in practice only incomplete information is available and could reflect sensor failure or practical restrictions on sensor placement, the availability of an extensive data set used in this study allowed some preliminary investigation into several hypothetical situations. In all the cases considered, the target of the optimization simulations was to arrive at a refined sensor array that would meet the single or multiple measurement objectives.

\section{NUMERICAL SIMULATIONS}

\subsection{Slender structure model particulars}

The basic cylinder configuration from the experimental data is illustrated in Fig. 3. The test cylinder has a length to diameter ratio of 263 and its response was resolved at $25(\mathrm{~N}=25)$ 


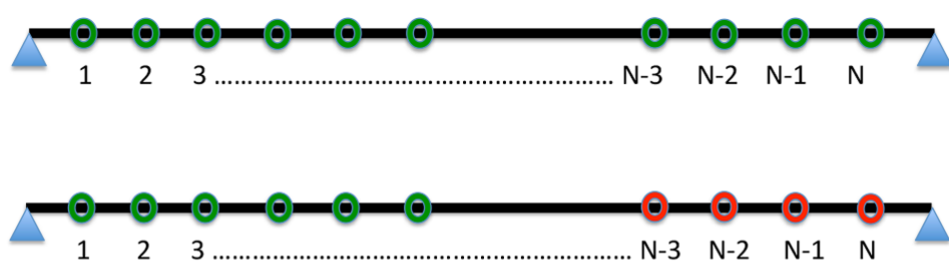

- Active sensor

- No sensor information

Figure 3: Unrestricted and restricted sensor configurations for pin-pined beam models of a slender structure.

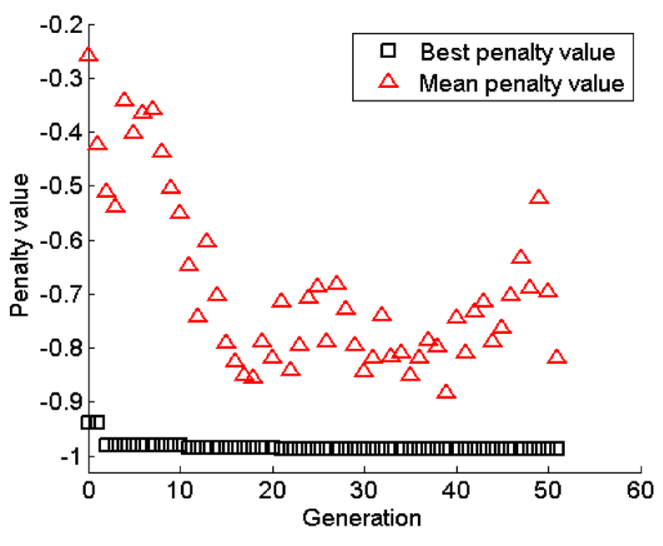

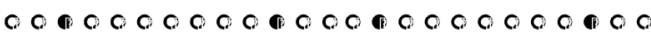

Figure 4: A typical Genetic Algorithm convergence pattern based upon targeting the 4th mode as the single objective and the final the resulting sensor location indicated with solid circles.

uniformly spaced sensor locations. The cylinder experiments that had a constant diameter were selected for this study, as their response behavior is expected to be more reflective of a wider range of structural engineering applications. The first configuration is used as the base case to explore optimized sensor locations for specified single and multiple objectives. The second configuration simulates a scenario where the failure of the sensors occurs or inability to locate sensors occurs. These are referred to the unrestricted and restricted configurations.

\subsection{Sensor number optimization for single and multiple objectives}

Two examples are considered in this section. The first simulation demonstrates the genetic algorithm solution to finding the optimal solution to the single objective defined as the accurate evaluation of 4th mode of vibration from a potential array of twenty-five equally spaced sensors. The solution presented in Fig. 4 illustrates the nature of the Genetic Algorithm solu- 

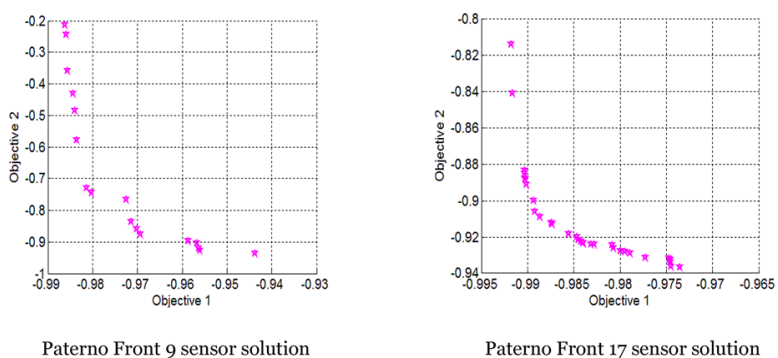

Figure 5: A Genetic Algorithm Paterno Front approach when two objective functions are specified.

tion convergence and divergence as the number of generations' increases even though the penalty function value is minimized. The penalty function here is defined so that value of the modal assurance criteria is minimized. The resulting optimized solution for accurately obtaining the 4th mode is a symmetric solution positioning the sensors at locations $3,11,15$ and 23 as illustrated in Fig. 4. The idea of symmetry will be utilized in a later example resolving evaluation of information from a restricted sensor configuration.

The second example seeks to find the optimal sensor configurations to accurately measure the 7th and 9th modes of the beam response. This is an example of the genetic algorithm solution with multiple objectives. Here a form of a Paterno Fronts representation presented in Fig. 5 illustrates the minimization of the penalty functions for each mode of interest [12]. In this case there is no simple solution and the number of sensors must be increased in order to accurately recover both modes. A comparison of the figures illustrates fewer sensors are needed to recover the 7th mode and that the accurate recovery of the 9th mode is more difficult as convergence is somewhat slower as indicated with the values of the penalty function as compared to those of the 7th mode. This example illustrates the difficulty in optimizing sensor arrays to recover higher modes of vibration.

\subsection{Incomplete sensor information}

Another practical challenge is to recover modes of interest when incomplete information from a sensor array is all that is available for analysis. The restriction of sensor information from an array could be the result of single or multiple sensor failures, limitations on the ability to place sensors on the structure for various reasons or perhaps because of other constraints. In this example, the recovery of the 5th mode is attempted with the four rightmost sensors unable to provide any information, as graphically illustrated earlier in Fig. 3. Thus, only 21 of the 25 sensors are available to provide information for the genetic algorithm to be utilized in the optimization process. The results of the modal resolution are presented in Fig. 6. The dashed blue line reflects the correct mode shape based upon the use of all information from the 25 unrestricted sensor configuration and is noted in the graph as the non-restricted case. The solid red line represents the resolution of the mode shape obtained for the restricted sensor configuration and the distortion of the peaks of the mode shape are constant while the distortion of the troughs are most notable closest to the sensor that are unavailable. There is also a noticeable phase shift at the opposite end of the restriction. The black dash-dotted line reflects the modified approach utilizing information on symmetry of the mode shape. It generally provides a 


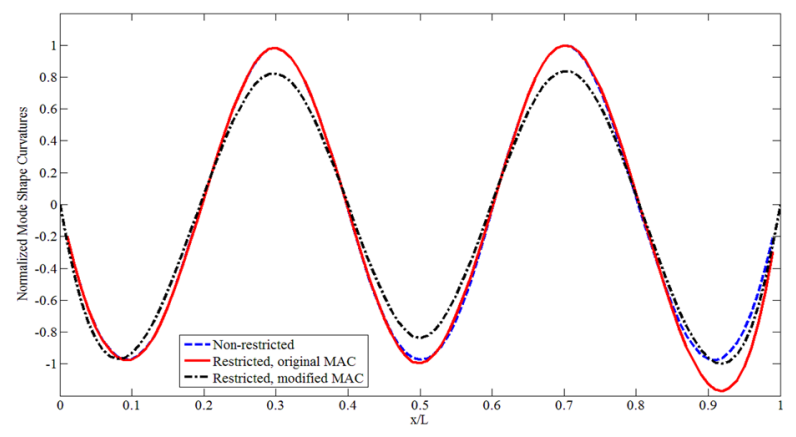

Figure 6: Mode shape comparisons with symmetry adjustment for the 5th mode with four sensors at the right end of the beam unavailable.

much improved amplitude solution with the exceptions of reduced trough amplitude at the beam center and a slight phase shift at the rightmost end where there is no sensor information.

\section{SUMMARY AND CONCLUSION}

One approach for guiding the optimization strategy regarding placement of sensors for the laboratory or field-testing of slender structural members was presented. The approach is independent of sensor type, arrangement or spacing. The framework was envisioned as consisting of an optimization domain that encompasses an analytical domain where the input to the optimization process is data that could be numerically simulated or actual experimental measurements. In this study data from sensors that were equally spaced was used. The methodology was used to search for the optimal number and location of sensors needed to meet specific modal measurement objectives. A Genetic Algorithm approach was adopted as the optimization method and the time domain decomposition (TDD) method that was integrated with the modal assurance criteria was used to evaluate the modal properties from different sensor combinations during the optimization process. The TDD method was selected as it is effective in recovering mode shapes, damping characteristics and modal contribution factors for data regardless of sensor spacing. The Modal Assurance Criterion was used as a measure to assess the convergence of the various sensor combinations in meeting single or multiple objectives. The Genetic Algorithm used in this study was modified to accelerate the solutions and to take advantage of mode symmetry [12]. The basic framework of the analysis, optimization domain components presented in Fig. 2, are quite general methods other than those selected for this research study could be used.

Data recorded from an experiment investigating the flow-induced vibration of a slender structural member was used in this optimization study. The resolution of the transverse motion response measurements provided the opportunity to explore the specification of a single objective, multiple objective and restriction scenarios and consequently the presentation of examples with increasing complexity. The first illustrative example dealt with the definition of a single objective to accurately assess a single response mode. The symmetry of the resulting minimal sensor array suggested that with additional insight symmetry might be exploited to further reduce the number of sensors required. Structural response may be dominated by a single mode but higher modes are present and also contribute to the response behavior. To investigate the nature of sensor optimization issues for multi-mode response 
behavior, two objective optimization targeting higher modes case was pursued and the use of a graphical variation of the Paterno Front Method was illustrated. The minimization of the penalty function for the modes selected was shown to be at issue and resulted in the introduction of many more sensors than perhaps desired. The third example explored the consequence of restricting sensor information at one end of the structure and the distortion of the single objective mode shape specified that was recovered from with incomplete information was illustrated. A modification to the optimization procedure recognizing the symmetry of mode shapes was introduced and produced a notable improvement. Further investigation of these preliminary findings is ongoing.

\section{ACKNOWLEDGEMENTS}

The legacy of Professor Connor I believe is best summed up as his deep intellectual pursuit of emerging topics in science and engineering that through his leadership have led to innovative thinking on course work development, graduate education and the professional practice of civil engineering. The number of graduate students and colleagues that he has influenced during his long career at MIT is both extraordinary and expletory.

It is with great admiration and friendship that his former colleague at MIT, Professor Kenneth F. Reinschmidt wrote:

'Unfortunately, in 50 years the stories have become less focused and definitely less meaningful to those who weren't there. (Although I do remember promising Jerry that I would advance the profession by not going into Engineering Mechanics). Although memories fade, it is the purpose of events like these today to revive and refresh these memories and our interpretations of what was going on and how we could improve it. The best summary I can think of I actually got from Chris Holley in a corridor discussion in Building 1: that we stayed at MIT not because MIT was ranked Number 1, but because MIT was where the action was. Looking back over those years I think we were privileged to be where the action was or close to it, pretty much all of the time. And wherever the action was, there was Jerry.'

His former student Dr David N. Ford, who was also unable to attend the retirement events, wrote:

'Jerry Connor saved me on my path to my $\mathrm{PhD}$. After passing my qualifying exam I unexpectedly found myself without an advisor and with few alternatives. This was one of my most anxious times. Professor Connor's stature in the department and reputation among the $\mathrm{PhD}$ students for fair and unbiased treatment led me to ask him for advice on how to proceed. He calmed my fears and pointed me to parts of MIT outside the Civil Engineering Department and in the direction that would (eventually) lead to the successful completion of my degree. Despite the fact that I was not, and would never be, focused on structures, he also accepted the role of chair of my committee and shepherded me through the remainder of my work at MIT. Professor Connor's unselfish service to me as a PhD student when I needed it most is part of the best of what MIT is and can be. I am deeply indebted and grateful.'

Finally, I wish to personally express my thanks to Professor Connor for our many memorable discussions over the years. Also, I would be remiss if I didn't express the sincere appreciation of Mary and our family to both Jerry and his wife Barbara for their many kindnesses, down to earth exchanges and the fond memories of just plain fun we have experienced together. 


\section{REFERENCES}

[1] Winston, P.H., Artificial Intelligence, 2nd edn., Addison-Wesley Publishing Company, Inc.: USA, 1984.

[2] Minsky, M. \& Papert, S., Preceptrons: Expanded Edition, MIT Press: Cambridge, MA, 1988.

[3] Abelson, H., Sussman, G.J. \& Sussman, J., Structure and Interpretation of Computer Programs, MIT Press: Cambridge, MA, 1985. doi: http://dx.doi.org/10.2307/3679579

[4] Connor, J.J., Niedzwecki, J.M. \& Guo, R.S., Intelligent mesh design for discrete element analysis, Artificial Intelligence in Engineering Conference, MIT: Cambridge, MA, 1987.

[5] Chehayeb, F., Niedzwecki, J.M., \& Connor, J.J., A knowledge based approach to innovative design. Artificial Intelligence in Engineering Conference, MIT: Cambridge, MA, 1987. doi: http://dx.doi.org/10.1016/0954-1810(87)90170-1

[6] Chehayeb, F., Connor, J.J. \& Niedzwecki, J.M., Innovative engineering design. Microcomputers in Civil Engineering, 4(1), pp. 1-9, 1989. doi: http://dx.doi. org/10.1111/j.1467-8667.1989.tb00001.x

[7] Niedzwecki, J.M. \& Earles, J., Strategy for design of intelligent tutoring systems. ASCE Journal of Computing in Civil Engineering, 5(2), pp. 211-229, 1991. doi: http://dx.doi. org/10.1061/(ASCE)0887-3801(1991)5:2(211)

[8] Niedzwecki, J.M. \& Rijken, O.R., Preliminary design of Tension Leg Platforms using a knowledge based approach. ASCE Ocean's V, 1, pp. 288-293, 1992.

[9] Connor, J.J. \& Faraji, S., Fundamentals of Structural Engineering, Springer International Publishing: New York, 2013.

[10] Connor, J.J. \& LaFlamme, S., Structural Motion Engineering, Springer International Publishing: New York, 2014.

[11] Coley, D.A., An Introduction to Genetic Algorithms for Scientists and Engineers, World Scientific: New Jersey, 2005.

[12] Fang, S.M., Structural Response Evaluation Using Non-uniform Sensor Arrays, Dissertation, Zachry Department of Civil Engineering, Texas A\&M University, College Station, TX, 2013.

[13] Fu, S., Ren, T., Li, R. \& Wang, X., Experimental investigation on VIV of the flexible model under full scale Re number. Proc. Offshore Mechanics and Arctic Engineering, No. 49042, 2011. doi: http://dx.doi.org/10.1115/OMAE2011-49042

[14] Li, L., Fu, S., Yang, J., \& Ren, T., Experimental investigation on VIV of risers with staggered buoyancy. Proc. Offshore Mechanics and Arctic Engineering, No. 49046, 2011. doi: http://dx.doi.org/10.1115/omae2011-49046

[15] Niedzwecki, J.M. \& Fang, S.M., Suppression of flow-induced vibrations using ribbon fairings. Journal of Computational Methods and Experimental Measurements, 1(4), pp. 395-405, 2013. doi: http://dx.doi.org/10.2495/cmem-v1-n4-395-405

[16] Fang, S.M. \& Niedzwecki, J.M., Comparison of airfoil and ribbon fairings for suppression of flow-induced vibrations. Journal of Computational Methods and Experimental Measurements, 2(1), pp. 30-45, 2014. doi: http://dx.doi.org/10.2495/cmem-v2n1-30-45

[17] Fang, S.M., Niedzwecki, J.M., Fu, S., Li, R. \& Yang, J., VIV response of a flexible cylinder with varied coverage by buoyancy elements and helical strakes. Marine Structures, 39, pp. 70-89, 2014. doi: http://dx.doi.org/10.1016/j.marstruc.2014.06.004

[18] Niedzwecki, J.M. \& Fang, S.M., Hydrodynamic response of ribbon fairing. Proc. Oceans 2013 MTS/IEEE, San Diego, CA, 2013. 